$11-1-2020$

\title{
Perceptions and use of non-pneumatic anti-shock garments for management of postpartum hemorrhage in Malawi
}

Breakthrough RESEARCH

Follow this and additional works at: https://knowledgecommons.popcouncil.org/departments_sbsr-rh How does access to this work benefit you? Let us know!

\section{Recommended Citation}

Breakthrough RESEARCH. 2020. "Perceptions and use of non-pneumatic anti-shock garments for management of postpartum hemorrhage in Malawi," APPHC Case Study. Washington, DC: Population Council. 


\section{Perceptions and use of non-pneumatic anti-shock garments for management of postpartum hemorrhage in Malawi}

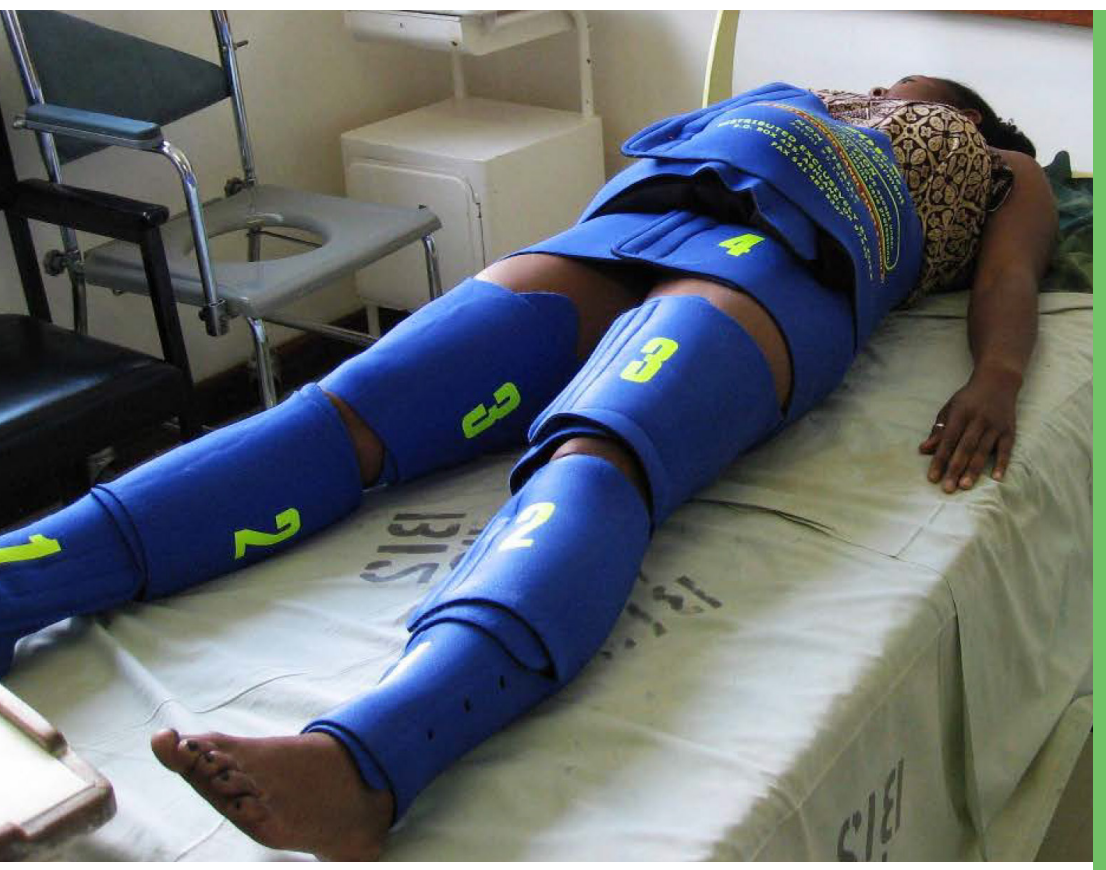

The non-pneumatic anti-shock garment (NASG) is a simple, neoprene and Velcro ${ }^{\mathrm{TM}}$ device that can be used to treat shock, resuscitate, stabilize, and prevent further bleeding in women experiencing postpartum hemorrhage (PPH)-a valuable tool to reduce PPH related deaths and severe morbidities. ${ }^{1-3}$ NASG use is recommended "in settings where delays in management of PPH are common, particularly where constraints to offer blood products and definitive treatment exist. NASG is an intervention that should be considered as a policy option while the standard conditions for care are being optimized." ${ }^{3}$

\section{KEY POINTS}

Providers recognize the importance of the NASG in management of PPH.

Provider knowledge and reports of receiving training on the NASG were generally low.

\section{RECOMMENDATIONS}

Continue to incorporate how and when to use the NASG into training for maternal health providers including during simulation exercises to build capacity and confidence to use them.

Build connections between hospitals that are confident in using the NASG with providers from lower level facilities to enable continued training on the NASG in Malawi.

Increase facility access to NASG at primary and secondary levels of the health system, especially at larger primary health centers (PHCs) with frequent deliveries and remote facilities.

Improve the quality and use of the monitoring system for NASG use to ensure NASGs are returned or replaced if women are referred wearing them, so that all maternity units have NASGs available at all times.
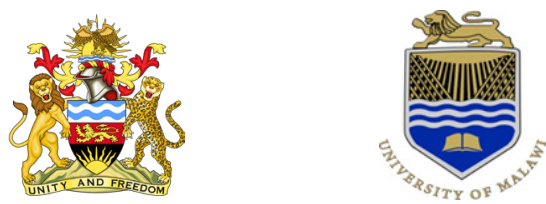
The NASG has been adopted and introduced by the Ministry of Health \& Population (MoH) in Malawi. Given that challenges in Malawi's patient referral and transport system often cause delays in care, NASGs are a potentially important tool for stabilizing women with PPH during delays in transport and receiving appropriate care.

This brief describes activities that are part of a larger portfolio of research led by the APPHC Partnership ${ }^{\text {a }}$ on prevention, detection, and management of $\mathrm{PPH}$, which continues to be the biggest threat to childbearing women in Malawi. ${ }^{4}$ Scoping activities, stakeholder consultations, and a formative assessment were conducted between April 2019 and March 2020 to understand key implementation barriers, bottlenecks and opportunities for improved prevention, early detection, and treatment of PPH in Malawi. The aims of this case study are to: 1 ) understand providers' knowledge, training, perceptions, and use of the NASG; and 2) identify appropriate implementation strategies to improve use of NASG for management of women with PPH in Malawi.

\section{Methods}

Scoping activities conducted by the APPHC Partnership in 2019, including visits to different levels of health facilities, highlighted the variable use of the NASG for PPH. National guidelines for PPH prevention and management were also reviewed to assess whether the NASG was included in PPH management guidelines and protocols. A mixed method formative study was conducted in the Lilongwe, Balaka, Zomba, and Dowa districts of Malawi. Quantitative interviews incorporated questions on use of NASGs including vignettes, which asked providers to describe the actions they would take in the case of PPH, to assess their applied knowledge of PPH management. Facility inventories assessed the number of NASGs in use at each facility. Lastly, inpatient record reviews were conducted to assess PPH management practices including use of the NASG. Qualitative interviews were conducted with providers to discuss NASGs, their introduction, training received, experience of use, challenges encountered, and how they have been used to mitigate morbidity and mortality resulting from PPH in Malawi.

\footnotetext{
The Advancing Postpartum Hemorrhage Care (APPHC) Partnership between Breakthrough RESEARCH and USAID's Health Evaluation and Applied Research Development (HEARD) Project, and the implementing partner Kamuzu College of Nursing under the University of Malawi, conducted formative research to understand key implementation barriers bottlenecks and opportunities for improved postnatal surveillance, prevention, and treatment of PPH in Malawi.
}

\section{Findings}

In 2015, the MoH published its Integrated Maternal and Neonatal Participants Manual but did not include a reference to NASGs for managing PPH in the guidelines. However, in 2018, the $\mathrm{RH}$ Directorate at the $\mathrm{MoH}$ developed obstetric management protocols for both hospitals and health centers with the following general management for PPH clearly stated:

- Call for help.

- Explain condition to the woman.

- Apply the anti-shock garment (if available and the provider trained).

\section{Availability of NASGs at facilities}

Among the study facilities ( $n=25), 48$ percent had one or more NASGs; 28 percent had one and 20 percent had two. Of the five facilities that had two NASGs, four were using both. All three district hospitals, eight community hospitals (80\%), and just one of the primary health centers (PHCS) had at least one NASG available (see Figure 1). The qualitative data also suggested a lack of widespread NASG availability.

\section{FIGURE 1 AVAILABILITY OF AT LEAST ONE NASG BY FACILITY TYPE IN SELECTED FACILITIES IN MALAWI}

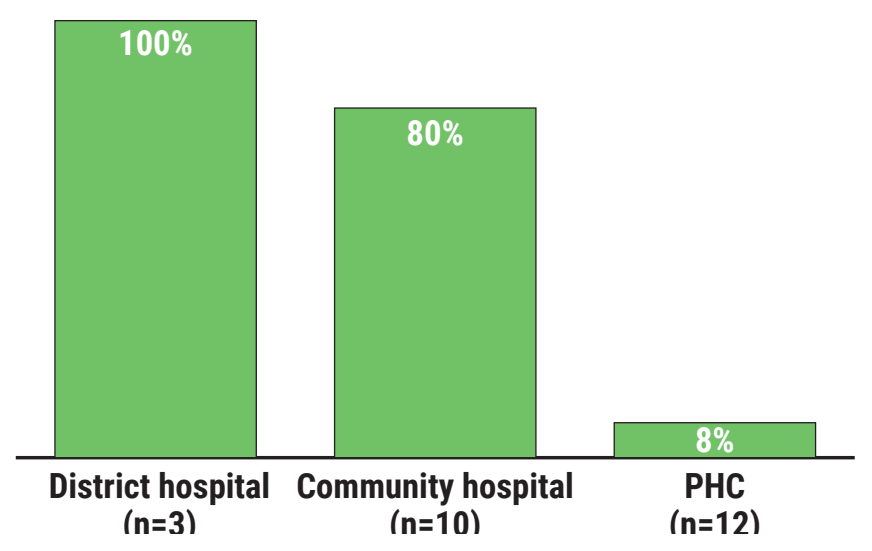

\section{I have no idea about what NASG is and I have never heard about it and we don't even have one at this facility.}

-IDI, Community midwife, Balaka District 
So far here it has not yet been used but we have it. It's there in our office but eh it's a long time since I learnt that. But it's what l've learnt that it's a good thing.

-IDI, Registered nurse midwife, Lilongwe District

Additionally, a major concern and apparent underlying reason for non-use of a NASGby providers was that a facility might only have one NASG - which might not be returned to the facility if a woman is referred wearing it:

And you know the challenge is this because...I will be alone here and after having PPH here that patient I'm supposed to send to [the district hospital]. Now who will bring back that NASG?

-IDI, Nurse midwife, Lilongwe District

\section{NASG awareness, training, and experience among providers}

In addition to a lack of NASG availability, providers also noted a lack of knowledge, training, and experience using the NASG.

2 The major challenges are lack of resources and knowledge gap. For example, we only have one NASG and again midwives do not know how to apply NASG.
Of 288 providers interviewed, 13.5 percent reported receiving NASG training in the three months preceding the survey. This was higher among providers at district hospitals $(19 \%)$ than community hospitals $(15 \%)$ or PHCs (8\%), as shown in Figure 2a. Eleven percent reported using a NASG in the previous three months. As might be expected, providers who said they had received training on how to use the NASG reported using it. In addition, providers at district hospitals were most likely to report use of NASGs $(18 \%)$ and were most often used in Dowa district (21\%) (Figure 2a and 2b).

When responding to $\mathrm{PPH}$ management vignettes, just under one quarter (24\%) of providers mentioned the NASG for PPH management. While there was little variation by facility type, providers in Dowa were most likely to mention the NASG (31\%), while only 19 percent did so in Lilongwe (Figure 2b).

Reviews of 177 women's inpatient records of PPH showed that a NASG was used in only 21 instances (12\%). A

NASG was most often used at district hospital level with majority documented in Balaka district, suggesting in most instances, the NASG was used for stabilization and management of PPH as opposed to stabilizing women for transfer.

Overall, the qualitative data also showed mixed levels of knowledge, training, and experience on how to use the NASG across the different levels of the health system:

-IDI, Nurse midwife, Dowa District

FIGURE 2 NASG AWARENESS, RECENT TRAINING AND USE AMONG PROVIDERS IN MALAWI

a. By facility type

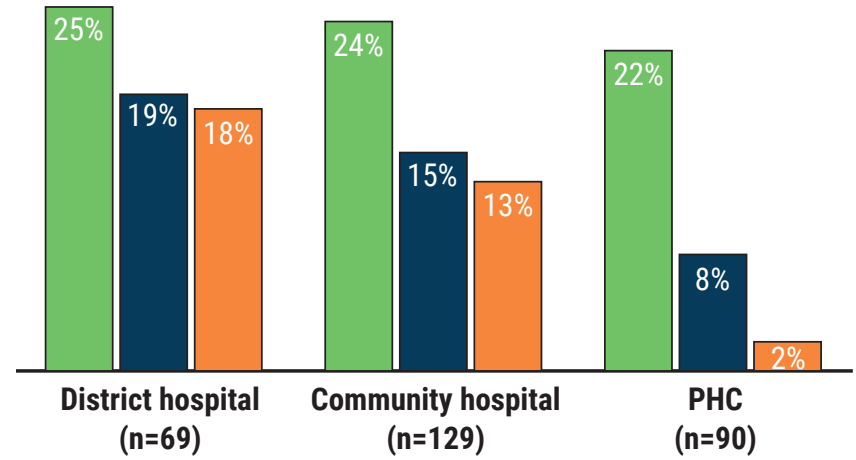

b. By district

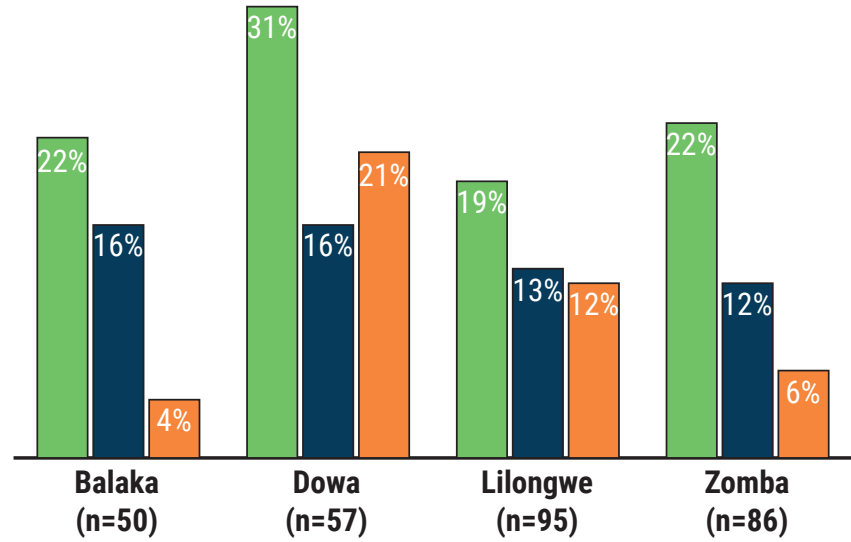

Mentioned during vignette

Recent training

Recent use 
NASG is a very good device and I have seen patients benefiting from it, not necessarily here...but also my previous workplace.... That's where it was frequently used other than here. For the past two years I have been here NASG was used only once.

-IDI, Nurse midwife, Dowa District

It is good though as of now I don't have much experience about it. How it is used, and how it controls. Until I know how it works and how they use and how it controls bleeding then I will be very happy.

-IDI, Nurse midwife, Lilongwe District

I have used the NASG once. We had a patient who developed PPH we did all interventions but the bleeding could not stop the patient started showing signs of shock so like dizziness and postural hypotension so we applied the NASG on the patient.

-IDI, Nurse midwife, Lilongwe District

Across all health facility levels, when asked how to improve the use of NASG, providers noted the lack of training as the main barrier to use:

The most basic thing is to train all staff; all midwives should be competent enough to use NASG and be able to know which patient is eligible for NASG, because it's not every mother who has lost blood can be applied NASG.

-IDI, Nurse midwife, Dowa District

\section{Discussion}

NASGs are an essential part of a continuum of care for PPH; they are not designed as a stand-alone intervention, ${ }^{5}$ but, when used as part of a package of interventions, women appear to have improved outcomes.

Even though the use of the NASG is recommended by $\mathrm{WHO}, \mathrm{FIGO}$, and $\mathrm{Malawi} \mathrm{MoH}$ to manage $\mathrm{PPH}$, findings from this mixed method case study showed knowledge, training, and recent use of the NASG is low. This is true across different health facility levels.

It is apparent from our findings that only around 1 in 10 providers interviewed had used one in the three months preceding the survey-and most of these were in hospitals. Few had received training recently and it was not clear if they had also been trained in methods of cleaning and storage. This was especially true for PHCs, where only 2 percent of providers had recently used a NASG for PPH management. While non-use of the NASG may be largely due to infrequent cases of PPH (especially at lower level facilities) as well as lack of inventory and training, providers also reported concern that women would be referred to a hospital while wearing a NASG, and the garment would not be returned to the facility. In 2016-2017 another project trained "field experts" on the indications for NASG use, proper application technique and removal, and method of cleaning and storage. While many demonstrated their skills and felt confident in using the NASG it is not clear how many additional providers were trained and at what level. ${ }^{6}$

Based on these findings, the following recommendations are made to improve knowledge and use of the NASG in PPH management in Malawi.

\section{Recommendations for increasing use of the NASG for PPH management}

- Continue to incorporate how and when to use the NASG into training for maternal health providers including during simulation exercises to build capacity and confidence to use them.

- Build connections between hospitals that are confident in using the NASG with providers from lower level facilities to enable continued training on the NASG in Malawi.

- Increase facility access to NASGs at primary and secondary levels of the health system, especially at larger PHCs with frequent deliveries and remote facilities.

- Improve the quality and use of the monitoring system for NASG use to ensure NASGs are returned or replaced if women are referred wearing them, so that all maternity units have NASGs available at all times. 


\section{References}

1. Safe Motherhood Program. 2009. "About NASG." San Francisco, CA: Bixby Center for Global Reproductive Health, University of California San Francisco.

2. World Health Organization. 2012. "WHO recommendation on the use of non-pneumatic anti-shock garments for the treatment of postpartum haemorrhage." Geneva: World Health Organization.

3. Pileggi-Castro, C. et al. 2015. "Non-pneumatic anti-shock garment for improving maternal survival following severe postpartum haemorrhage: a systematic review," Reproductive Health 12(1): 28. doi: 10.1186/s12978-015-0012-0

4. Ministry of Health. 2015. Malawi Emergency Obstetric and Newborn Care Needs Assessment, 2014. Lilongwe: Ministry of Health.

5. FIGO Safe Motherhood and Newborn Health Committee. 2014. "Non-pneumatic anti-shock garment to stabilize women with hypovolemic shock secondary to obstetric hemorrhage," International Journal of Gynecology \& Obstetrics 128(3): 194-195. doi: 10.1016/j. ijgo.2014.10.014

6. Maternova. 2019. "AIM skills lab training in Malawi using NASG," Maternova, 3 July.
This work is part of the Advancing Postpartum Hemorrhage Care (APPHC) partnership supported by USAID and led by the Breakthrough RESEARCH Project and the Health Evaluation and Applied Research Development (HEARD) Project. The APPHC partnership generates and tests solutions to address key implementation barriers for PPH prevention and treatment and contributes to the effective implementation of interventions, strategies, and innovations for PPH in Madagascar and Malawi.

https://www.respectfulcareresources.com/apphc

\section{Acknowledgments}

This case study describes work led by the Population Council for the APPHC activity in Malawi. We would like to thank Mrs. Fannie Kachale (Director of Reproductive Health Services, MoH), Dr. Henry Phiri (Deputy Director of Reproductive Health Services, MoH); Drs. Abigail Kazembe and Martha Kamanga (KCN); and Elizabeth Tobey, Brady Zieman, and Dr. Charlotte Warren (Population Council), whose support, leadership, and contributions have made this work possible.

\section{Suggested citation}

Breakthrough RESEARCH. 2020. "Perceptions and use of nonpneumatic anti-shock garments for management of postpartum hemorrhage in Malawi," APPHC Case Study. Washington DC: Population Council.

Front cover photo credit: https://doi.org/10.1371/journal. pone.0076477.g001 (CC BY 2.0)

(C)2020 The Population Council. All rights reserved.

\section{Breakthrough RESEARCH}

Population Council

4301 Connecticut Ave., NW

Suite 280

Washington, DC 20008

+12022379400

breakthroughactionandresearch.org

BreakthroughResearch@popcouncil.org
USAID's Health Evaluation and Applied Research Development

URC

5404 Wisconsin Ave

Suite 800

Chevy Chase, MD 20815

www.heardproject.org

heard@urc-chs.com

\section{USAID}

This document is made possible by the generous support of the American people through the United States Agency for International Development (USAID) under cooperative agreements Breakthrough RESEARCH (AID-OAA-A-17-00018) and USAID's Health Evaluation and Applied Research Development Project (HEARD) (AID-OAA-A-17-00002). The contents of this document are the sole responsibility of Breakthrough RESEARCH, Population Council, and University Research Co., LLC and do not necessarily reflect the views of USAID or the United States Government.
USAID'S HEALTH EVALUATION AND APPLIED RESEARCH DEVELOPMENT (HEARD) PROJECT

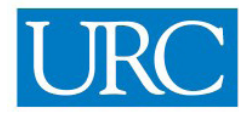

USAID's Health Evaluation and Applied Research Development (HEARD) project leverages a global partnership of more than 30 institutions to generate, synthesize, and use evidence to improve the implementation of policies and programs related to USAID priority areas, and crucial for improving health and development in low and middle-income countries.

\section{peounnox COUNCIL \\ Ideas. Evidence. Impact.}

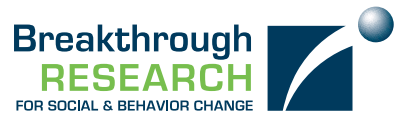

Breakthrough RESEARCH catalyzes SBC by conducting state-of-the-art research and evaluation and promoting evidence-based solutions to improve health and development programs around the world. Breakthrough RESEARCH is a consortium led by the Population Council in partnership with Avenir Health, ideas42, Institute for Reproductive Health at Georgetown University, Population Reference Bureau, and Tulane University. 